\title{
Optimization of Deep Oxidative Desulfurization Process Using Ionic Liquid and Potassium Monopersulfate
}

\author{
Yinke Zhang, ${ }^{1}$ Hang Xu $\mathbb{D},{ }^{2}$ Mengfan Jia, ${ }^{1}$ Zhuang Liu, ${ }^{1}$ and Deqiang Qu ${ }^{1}$ \\ ${ }^{1}$ School of Mathematics and Statistics, Henan University of Science and Technology, Luoyang 471023, China \\ ${ }^{2}$ School of Chemical Engineering and Pharmaceutics, Henan University of Science and Technology, Luoyang 471023, China \\ Correspondence should be addressed to Hang Xu; xhinbj@126.com
}

Received 5 December 2017; Revised 15 February 2018; Accepted 3 April 2018; Published 9 May 2018

Academic Editor: Tingyue Gu

Copyright (c) 2018 Yinke Zhang et al. This is an open access article distributed under the Creative Commons Attribution License, which permits unrestricted use, distribution, and reproduction in any medium, provided the original work is properly cited.

\begin{abstract}
Response surface methodology (RSM) was selected to optimize a desulfurization process with metal based ionic liquids $\left([\mathrm{Bmim}] \mathrm{Cl} / \mathrm{CoCl}_{2}\right)$ and potassium monopersulfate (PMS) together to remove benzothiophene (BT) from octane (simulating oil). The four experimental conditions of PMS dosage, $[\mathrm{Bmim}] \mathrm{Cl} / \mathrm{CoCl}_{2}$ dosage, temperature, and reaction time were investigated. The results showed that the quadratic relationship was built up between BT removal and four experimental variables with 0.9898 fitting coefficient. The optimal conditions were $1.6 \mathrm{~g}$ (20 wt\%) PMS solution, $3.2 \mathrm{~g}[\mathrm{Bmim}] \mathrm{Cl} / \mathrm{CoCl}_{2}, 46^{\circ} \mathrm{C}$, and $23 \mathrm{~min}$, which were obtained based on RSM and experimental results. Under the optimal condition, predicted sulfur removal rate and experimental sulfur removal rate were $96.7 \%$ and $95.4 \%$, respectively. The sequence of four experimental conditions on desulfurization followed the order temperature $>$ time $>[\mathrm{Bmim}] \mathrm{Cl} / \mathrm{CoCl}_{2}$ dosage $>$ PMS solution dosage.
\end{abstract}

\section{Introduction}

Over the past fifteen years, many developing countries have been suffering from severe haze which seriously affects people's health. One of the reasons is attributed to burning the sulfur-containing fuels and producing sulfur oxide which can form the haze and acid rain to pollute environment [1]. In order to control environmental pollution caused by sulfur oxide, almost all countries tightly limit the sulfur content in fuel oil. In 2016, China enacted the national law to control the sulfur content in fuel oil that must be less than $10 \mathrm{ppm}$, the same as USA and European countries [2].

In order to remove organic sulfur compound in oil, there are some effective technologies like hydrodesulfurization, adsorption, extraction, oxidation, and so on. Nowadays, ionic liquid attracts more attention in desulfurization field [3] because of its green solvent characteristics $[4,5]$ and catalytic activity. Zhang et al. [6] used [HDBN]Cl/ $\mathrm{ZnCl}_{2}$ ionic liquid and $\mathrm{H}_{2} \mathrm{O}_{2}$ to treat model oil and obtained $98.8 \%$ dibenzothiophene (DBT) removal. Safa et al. [7] used acidic ionic liquid loaded on silica gel as catalyst for desulfurization and $75.7 \%$ of sulfur removal of real diesel was obtained.
The response surface methodology (RSM) is a mathematical statistical method which is always applied to the process optimization. In conventional multifactor experiments, optimal process is usually carried out by varying one factor and fixing all other factors (i.e., one factor at a time). Alternatively, the RSM enables one to determine the influence of individual factors as well as their interactive influences with a limited number of planned experiments. Thus, the RSM is widely used in various industrial, agricultural, and other research fields [8].

Chen et al. [9] prepared $\left[\mathrm{C}_{4} \mathrm{mim}\right] \mathrm{Cl} / \mathrm{ZnCl}_{2}$ ionic liquid and used it to investigate the oxidative removal of sulfur compounds from diesel fuels, with $30 \mathrm{wt} \% \mathrm{H}_{2} \mathrm{O}_{2}$ solution as oxidant. In this study, the temperature, IL dosage, $\mathrm{H}_{2} \mathrm{O}_{2}$ dosage, and reaction time were investigated for desulfurization. Chi et al. [10] also synthesized a functional ionic liquid combined with $30 \mathrm{wt} \% \mathrm{H}_{2} \mathrm{O}_{2}$ solution for desulfurization and IL dosage, $\mathrm{H}_{2} \mathrm{O}_{2}$ dosage, temperature, and reaction time were investigated.

In this study, we prepared a functional $[\mathrm{Bmim}] \mathrm{Cl} / \mathrm{CoCl}_{2}$ ionic liquid (IL) with potassium monopersulfate (PMS) to remove benzothiophene (BT) from octane. The effects of 
four independent variables of PMS dosage, $[\mathrm{Bmim}] \mathrm{Cl} / \mathrm{CoCl}_{2}$, temperature, and reaction time for desulfurization were investigated based on response surface methodology (RSM) and real experiment.

\section{Experimental Methods}

2.1. Desulfurization Process. The $[\mathrm{Bmim}] \mathrm{Cl} / \mathrm{CoCl}_{2}$ ionic liquid (IL) was prepared by mixing $0.1 \mathrm{~mol} \mathrm{CoCl}_{2} \cdot 6 \mathrm{H}_{2} \mathrm{O}$ and 0.1 mol 1-n-butyl-3-methylimidazolium chloride $([\mathrm{Bmim}] \mathrm{Cl})$ at $110^{\circ} \mathrm{C}$ for $48 \mathrm{~h}$. The model oil was prepared by dissolving benzothiophene (BT) in octane maintaining sulfur content of $500 \mathrm{ppm}$. The desulfurization tests were carried out in a $40 \mathrm{~mL}$ two-necked flask equipped with a stirrer and a condenser. The desulfurization procedure was run as follows: firstly, $[\mathrm{Bmim}] \mathrm{Cl} / \mathrm{CoCl}_{2}$ and the simulating oil were added to the flask and the mixture was vigorously stirred for 30 min until the extraction equilibrium; secondly, $20 \mathrm{wt} \%$ PMS solution was added to the flask and quickly diffused into the ionic liquid phase. Subsequently, the mixture was vigorously stirred to complete the desulfurization reaction. All the chemicals in this study were purchased from Aladdin chemical reagent company. After reaction, aliquots of the upper phase (model fuel) were obtained. The remaining compounds were determined by GC (Agilent 7820A GC, USA) with a flame ionization detector (FID) and HP-1 capillary column (19091Z-443, $30 \mathrm{~m} \times 0.32 \mathrm{~mm} \times 0.25 \mathrm{um})$. The S-removal efficiency was calculated according to (1) as follows:

$$
\text { S-removal efficiency }(\%)=\left(1-\frac{[S]_{t}}{[S]_{0}}\right) \times 100,
$$

where $[S]_{0}$ was the initial S-content and $[S]_{t}$ was the S-content at time $t$.

2.2. Experimental Design for Desulfurization Process. Experimental design, mathematical modeling, and optimization process were performed using Design-Expert 8.0.6 software. Box-Behnken Design (BBD), one of the most common design methods, was used for the response surface methodology (PMS). The levels of the variables for desulfurization process are shown in Table 1 . The experimental data were analyzed by the response surface regression procedure to fit the following second-order polynomial model [11]:

$$
Y=\beta_{0}+\sum_{i=1}^{k} \beta_{i} X_{i}+\sum_{i=1}^{k} \beta_{i i} X_{i}^{2}+\sum_{i=1}^{k} \sum_{j=1}^{k} \beta_{i j} X_{i} X_{j}
$$

Here, $Y$ is the predicted response (sulfur removal, \%); $X_{i}$ and $X_{j}$ are variables (experimental conditions); $\beta_{0}$ is the constant coefficient; and $\beta_{i}$ is the coefficient that determines the influence of parameter $i$ in the response (linear term), which refers to the effect of the interaction among independent variables.

In this study, $[\mathrm{Bmim}] \mathrm{Cl} / \mathrm{CoCl}_{2}$ ionic liquid and $\mathrm{PMS}$ were investigated to remove benzothiophene (BT) from octane under different conditions such as initial PMS (20\% solution) (1-2 g), $[\mathrm{Bmim}] \mathrm{Cl} / \mathrm{CoCl}_{2}(2-4 \mathrm{~g})$, temperature $\left(40-60^{\circ} \mathrm{C}\right)$,
TABLE 1: Levels of the variables for BBD experimental design.

\begin{tabular}{lccccc}
\hline Variables & \multirow{2}{*}{ Code } & \multirow{2}{*}{ Unit } & \multicolumn{3}{c}{ Levels } \\
& & & -1 & 0 & 1 \\
\hline PMS & $A$ & $\mathrm{~g}$ & 1 & 1.5 & 2 \\
{$[\mathrm{BMIM}] \mathrm{Cl} / \mathrm{CoCl}_{2}$} & $B$ & $\mathrm{~g}$ & 2 & 3 & 4 \\
Temperature & $\mathrm{C}$ & ${ }^{\circ} \mathrm{C}$ & 40 & 50 & 60 \\
Time & $D$ & $\min$ & 5 & 15 & 25 \\
\hline
\end{tabular}

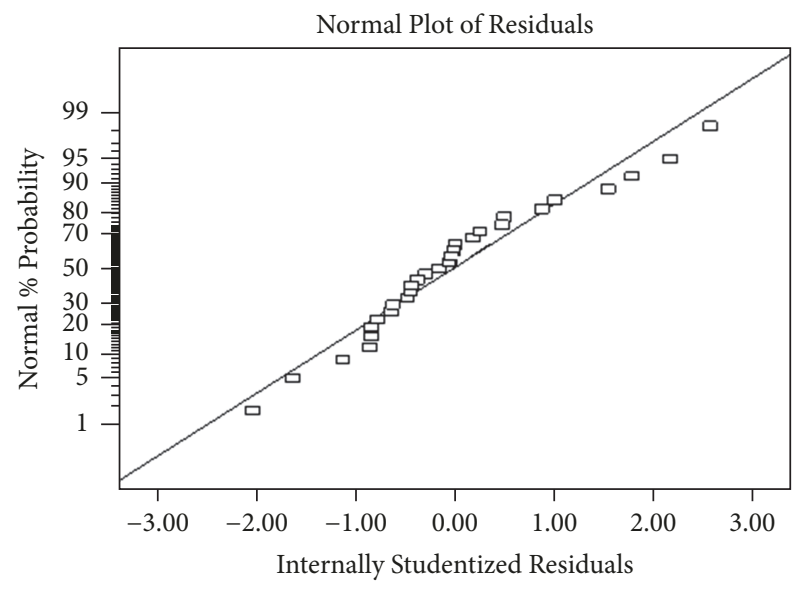

FIGURE 1: Normal probability of residuals.

and time (5-25 min). Based on Box-Behnken Design, the design matrix of coded values for the factors and the response in terms of sulfur removal for all 29 experimental runs are shown in Table 2, in which the amount of PMS, IL, temperature, and time are denoted as $A, B, C$, and $D$, respectively.

\section{Results and Discussion}

3.1. ANOVA Analysis. The relationship between S-removal and variables $(A, B, C$, and $D)$ was obtained and exhibited in (3) by Design-Expert 8.0 software calculation. The experimental results were verified by comparing them with the calculated data (i.e., the predicted values) which were shown in Table 2.

$$
\begin{aligned}
& \text { S-removal percentage } \\
& \begin{aligned}
= & 91.94+4.23 A+4.57 B-10.27 C+7.72 D \\
& -1.88 A B+5.07 A C+0.70 A D+1.35 B C \\
& +2.37 B D+0.075 C D-16.58 A^{2}-9.60 B^{2} \\
& -11.83 C^{2}-6.55 D^{2} .
\end{aligned}
\end{aligned}
$$

Figures 1 and 2 show the diagnostics of the fitted models in terms of comparison between the normality plot of residuals and the predicted and actual values. The residuals were defined as the differences between the experimental and predicted values. As can be seen from Figure 1, the residuals are in a normal distribution because a lot of data are near -1 
TABLE 2: Experimental design matrix and results for process optimization.

\begin{tabular}{|c|c|c|c|c|c|c|}
\hline Run & $\begin{array}{c}A \\
\mathrm{PMS} / \mathrm{g}\end{array}$ & $\begin{array}{c}B \\
\mathrm{IL} / \mathrm{g}\end{array}$ & $\begin{array}{c}\mathrm{C} \\
\mathrm{T} /{ }^{\circ} \mathrm{C} \\
\end{array}$ & $\begin{array}{c}D \\
\text { Time/min }\end{array}$ & $\begin{array}{c}\text { S-removal } \\
\text { Experimental results/\% }\end{array}$ & $\begin{array}{c}\text { S-removal } \\
\text { Predicted values/\% }\end{array}$ \\
\hline 1 & 1 & 2 & 50 & 15 & 54.3 & 55.1 \\
\hline 2 & 2 & 2 & 50 & 15 & 65.9 & 67.3 \\
\hline 3 & 1 & 4 & 50 & 15 & 68.6 & 68.0 \\
\hline 4 & 2 & 4 & 50 & 15 & 72.7 & 72.7 \\
\hline 5 & 1.5 & 3 & 40 & 5 & 75.6 & 76.2 \\
\hline 6 & 1.5 & 3 & 60 & 5 & 55.5 & 55.5 \\
\hline 7 & 1.5 & 3 & 40 & 25 & 90.7 & 91.5 \\
\hline 8 & 1.5 & 3 & 60 & 25 & 70.9 & 71.1 \\
\hline 9 & 1 & 3 & 50 & 5 & 57.2 & 57.6 \\
\hline 10 & 2 & 3 & 50 & 5 & 62.1 & 64.6 \\
\hline 11 & 1 & 3 & 50 & 25 & 72.7 & 71.6 \\
\hline 12 & 2 & 3 & 50 & 25 & 80.4 & 81.5 \\
\hline 13 & 1.5 & 2 & 40 & 15 & 76.6 & 77.6 \\
\hline 14 & 1.5 & 4 & 40 & 15 & 84.6 & 84.0 \\
\hline 15 & 1.5 & 2 & 60 & 15 & 52.3 & 54.3 \\
\hline 16 & 1.5 & 4 & 60 & 15 & 65.7 & 66.2 \\
\hline 17 & 1 & 3 & 40 & 15 & 74.1 & 74.6 \\
\hline 18 & 2 & 3 & 40 & 15 & 75.2 & 73.0 \\
\hline 19 & 1 & 3 & 60 & 15 & 43.9 & 44.0 \\
\hline 20 & 2 & 3 & 60 & 15 & 65.3 & 62.6 \\
\hline 21 & 1.5 & 2 & 50 & 5 & 69.1 & 65.9 \\
\hline 22 & 1.5 & 4 & 50 & 5 & 70.5 & 70.3 \\
\hline 23 & 1.5 & 2 & 50 & 25 & 78.5 & 76.6 \\
\hline 24 & 1.5 & 4 & 50 & 25 & 89.4 & 90.4 \\
\hline 25 & 1.5 & 3 & 50 & 15 & 91.9 & 91.9 \\
\hline 26 & 1.5 & 3 & 50 & 15 & 93.7 & 91.9 \\
\hline 27 & 1.5 & 3 & 50 & 15 & 92.4 & 91.9 \\
\hline 28 & 1.5 & 3 & 50 & 15 & 91.2 & 91.9 \\
\hline 29 & 1.5 & 3 & 50 & 15 & 90.7 & 91.9 \\
\hline
\end{tabular}

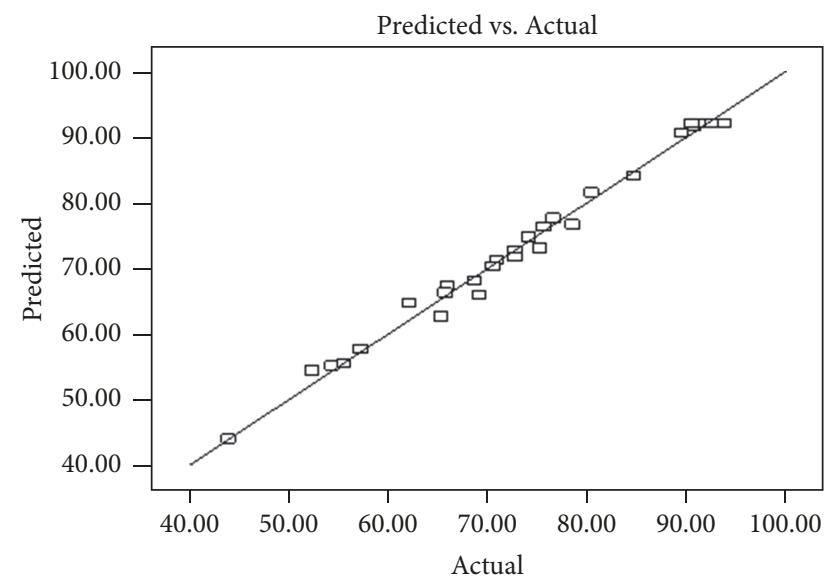

FIGURE 2: Plots of actual response versus predicted response.

to 1 of the $x$-axis. From Figure 2, all the data are near the line which meant the predicted values are close to experimental values, indicating that the model is successful in capturing the correlations between the influencing parameters and sulfur removal.

Table 3 presents the ANOVA results for response surface quadratic model. The significance of each coefficient is determined by $F$-value. For the operating parameters, values of "Prob $>F$ " are less than 0.05 , which indicates model term is significant. In this case, $A, B, C, D, A C, B D, A^{2}, B^{2}$, $C^{2}$, and $D^{2}$ are significant model terms, indicating that all the single parameters have a great impact on desulfurization. The $A C$ is significant which means that the interaction effect between PMS and temperature exhibits a great influence on desulfurization. The interaction between IL and time is also very strong impact on desulfurization because "Prob $>F$ " value of $\mathrm{BD}$ value is lower than $0.05[12,13]$. In contrast, $A B, A D, B C$, and $C D$ have negligible effect on the sulfur removal because the "Prob $>F$ " values are bigger than 0.05 . Based on the SS values of four conditions, the desulfurization efficiencies follow the order: temperature $>$ time $>$ IL $>$ PMS. The Lack of Fit is an important data to evaluate the reliability of the model. If the model is significant, it is not good for fit and needs to be adjusted. If the model is not significant, it is 
TABLE 3: Analysis of variance (ANOVA) for response surface quadratic model.

\begin{tabular}{|c|c|c|c|c|c|}
\hline Source & SS & $\mathrm{df}$ & MS & $F$ value & Prob. $>F$ \\
\hline Model & 5075.6 & 14 & 362.5 & 96.9 & $<0.0001$ \\
\hline$A$ & 215.1 & 1 & 215.1 & 57.5 & $<0.0001$ \\
\hline$B$ & 250.2 & 1 & 250.2 & 66.9 & $<0.0001$ \\
\hline C & 1264.9 & 1 & 1264.9 & 338.1 & $<0.0001$ \\
\hline$D$ & 714.6 & 1 & 714.6 & 191 & $<0.0001$ \\
\hline$A B$ & 14.1 & 1 & 14.1 & 3.76 & 0.0730 \\
\hline$A C$ & 103.0 & 1 & 103.0 & 27.5 & 0.0001 \\
\hline$A D$ & 2.0 & 1 & 2.0 & 0.52 & 0.4811 \\
\hline$B C$ & 7.3 & 1 & 7.3 & 1.95 & 0.1845 \\
\hline$B D$ & 22.6 & 1 & 22.6 & 6.03 & 0.0277 \\
\hline$C D$ & 0.02 & 1 & 0.02 & 0.006 & 0.9393 \\
\hline$A^{2}$ & 1782.8 & 1 & 1782.8 & 476.5 & $<0.0001$ \\
\hline$B^{2}$ & 598.2 & 1 & 598.2 & 159.8 & $<0.0001$ \\
\hline$C^{2}$ & 907.5 & 1 & 907.5 & 242.6 & $<0.0001$ \\
\hline$D^{2}$ & 278.6 & 1 & 278.6 & 74.5 & $<0.0001$ \\
\hline Residual & 52.4 & 14 & 3.7 & & \\
\hline Lack of fit & 46.5 & 10 & 4.7 & 3.13 & 0.1412 \\
\hline Pure error & 5.9 & 4 & 1.5 & & \\
\hline Total & 5128.0 & 28 & & & \\
\hline
\end{tabular}

${ }^{*} \mathrm{CV} \%=2.63 ; R^{2}=0.9898 ; \mathrm{Adj}-R^{2}=0.9796$.

simulated in an acceptable way. "Prob. $>F$ " is 0.1412 which implies that the "Lack of Fit" is not significant. The "Lack of Fit" could occur which is attributed to noise [14].

The correlation coefficient $\left(R^{2}\right)$ [15] can be used to describe degree of correlation between dependent variable (Sremoval) and independent variables $(A, B, C$, and $D)$, which is obtained by Design-Expert 8.0.6 software. The $R^{2}$ value in this study is 0.9898 , which indicates the correlation model (see (3)) is right. In addition, the $R^{2}$ value and adjusted$R^{2}$ value $(0.9796)$ are similar which means there is a little chance that nonsignificant term has been included in the model. The coefficient of variance $(\mathrm{CV})$ is another statistic to estimate the ratio of the standard deviation and the averages for observed results and measure the degree of variation for each observation. It also is a kind of evaluation profile of model reproducibility. As a general rule, a model can be considered reasonably reproducible when $\mathrm{CV}$ is not greater than $10 \%[15,16]$. The CV for this model is only $2.63 \%$, showing a good precision and reliability of the experimental runs.

3.2. Response Surface Plot and Contour Plot. The threedimensional response surface plot is useful to determine the individual and cumulative effects of one variable or the mutual interaction between two variables. The response surface analyzes the geometric nature of surface, the maxima and minima of the response, and the significance of the coefficients of the canonical equation [17]. The contour plots help to identify the type of interactions between tested variables. Each contour curve represents an infinite amount of sulfur removal under variation of two tested variables with other variables at the respective zero level.

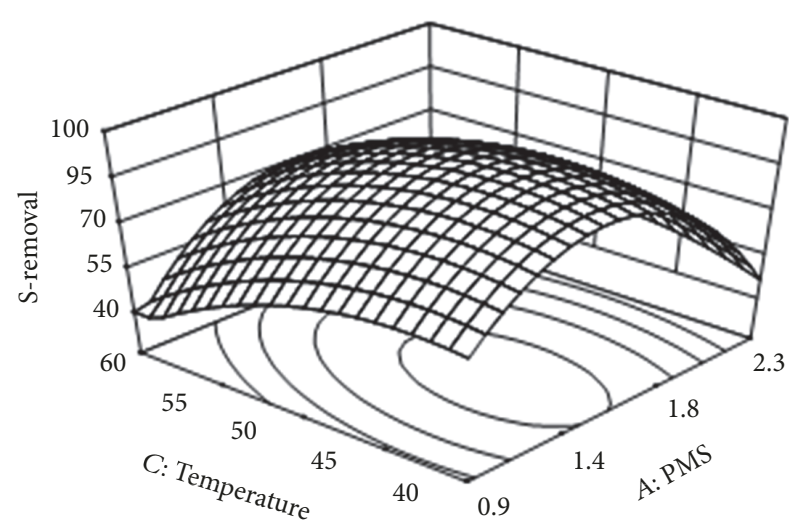

FIGURE 3: The response surface plot about effect of PMS and temperature interaction on S-removal.

Figures 3-6 show three-dimensional response surface plots and contour plots about the relationship between sulfur removal and four variables. From the ANOVA analysis, two experimental variables of PMS solution dosage $(A)$ and temperature $(C)$ could produce interaction effect on Sremoval. The ionic liquid $(B)$ and time $(D)$ together also exhibit interaction effect. So, Figure 3 shows the response surface plot which expresses the desulfurization effect of PMS solution dosage $(A)$ and temperature $(C)$ under zero level of ionic liquid $(B)$ and time $(D)$. Similarly, Figure 5 shows the response surface plot on desulfurization effects of ionic liquid $(B)$ and time $(D)$ under zero level of PMS solution dosage and temperature. Figures 4 and 6 show the contour plots.

It can be seen from Figure 3 that sulfur removal increases with the rise of PMS dosage. However, when the PMS 


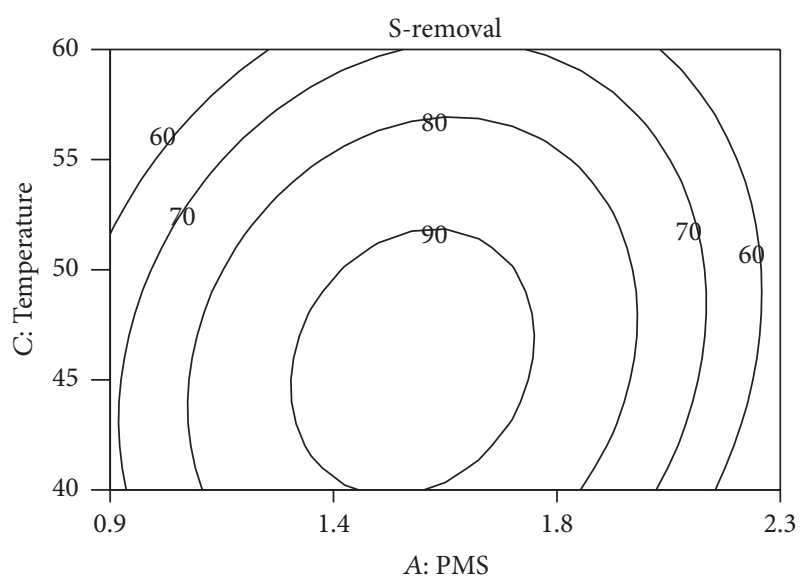

FIgURE 4: The contour plot about effect of PMS and temperature interaction on S-removal.

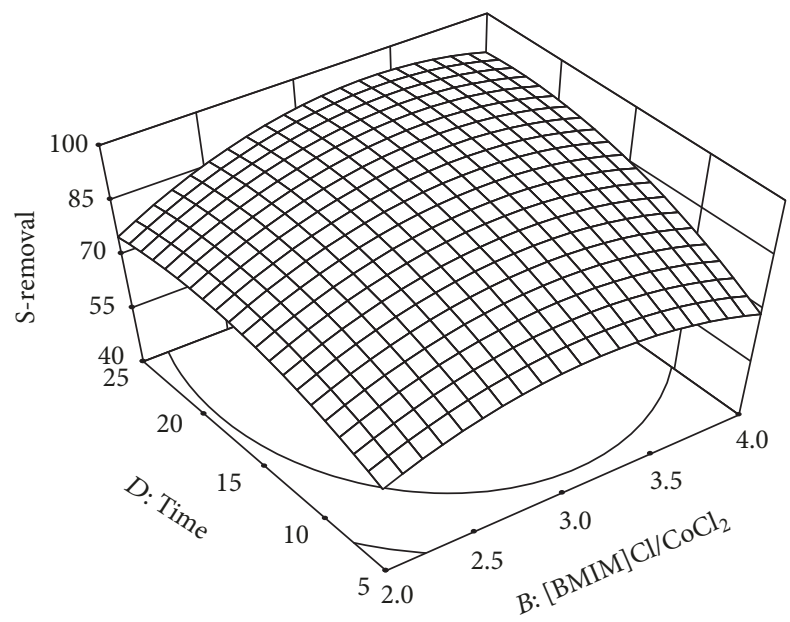

FIGURE 5: The response surface plot about effect of [BMIM]Cl/ $\mathrm{CoCl}_{2}$ dosage and time interaction on S-removal.

dosage is up to a certain value, continuing to increase the PMS dosage will inhibit the desulfurization process and result in sulfur removal down. The reason is that excess peroxosulphate $\left(\mathrm{HSO}_{5}{ }^{-}\right)$species could act as sulfate radical scavengers decreasing the desulfurization effectiveness [18]. From Figure 3, the temperature also has the optimal value. The reason is that high temperatures are not beneficial for sulfur removal because they negatively affect the catalytic oxidative and extraction processes. Thus, desulfurization is hindered at high temperatures since PMS is unstable and decomposes at temperatures as low as $65^{\circ} \mathrm{C}$. PMS loses its oxidative capacity above $60^{\circ} \mathrm{C}$. However, high temperatures help reduce the ionic liquid viscosity which is beneficial for achieving a good mixing between the ionic liquid and the fuel. At low temperatures, the viscosity of the ionic liquid is very high, thereby significantly hindering the mixing process between the ionic liquid and the fuel. From the contour plot (Figure 4), contour plot exhibits a closed oval contour curve which means the biggest sulfur removal is at the core of oval. If the temperature is fixed under a certain value,

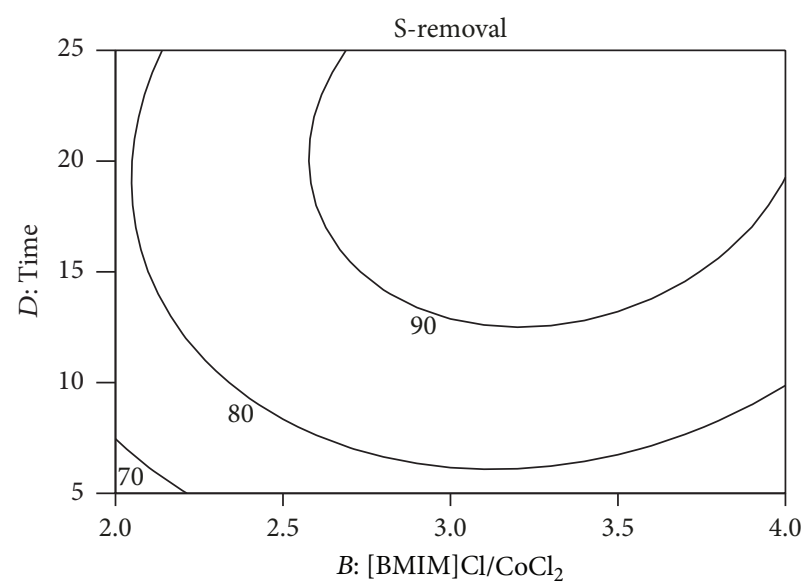

Figure 6: The contour plot about effect of [BMIM]Cl/ $\mathrm{CoCl}_{2}$ dosage and time interaction on S-removal.

S-removal exhibited a tendency of increase first and then decrease during the rise of PMS dosage. The same tendency also occurs during the rise of temperature under fixed PMS dosage value. Therefore, the PMS dosage and temperature exist the optimal values.

From Figure 5, it also can be seen that $[\mathrm{BMIM}] \mathrm{Cl} / \mathrm{CoCl}_{2}$ (IL) dosage has the optimal value. The reason is that at these high IL loading conditions, cobalt ions in ionic liquid phase might react with PMS to produce more sulfate radical species to oxidize BT molecules. Theoretically, the S-removal efficiency should increase with the IL dosage. However, if ionic liquid dosage exceeds the optimal value, excess cobalt ions can act as free radical scavengers consuming sulfate radicals and resulting in a poor desulfurization performance $[19,20]$. In Figure 6, the contour plot exhibits S-removal which expresses a tendency of first increase and then decrease during rise of IL dosage under fixed time. Reaction time is also an important condition that should be investigated. From Figure 5, the S-removal would have almost no change after a certain time under a fixed ionic liquid.

The optimal experimental conditions can be rapidly determined from the response surface plots under only 29 experiment points. The RSM is a convenient method to help us to search the optimal condition.

\subsection{Desulfurization Mechanism and Process Optimization.} The possible mechanism of desulfurization is that firstly BT is extracted by $[\mathrm{BMIM}] \mathrm{Cl} / \mathrm{CoCl}_{2}$ from octane phase to IL phase. Secondly, BT is oxidized in IL phase by sulfate radical which is from the reaction of $\mathrm{Co}^{2+}$ and PMS. The optimized conditions and predicted sulfur removal are shown in the Table 4. Using this optimized experimental condition, the experimental run for IL and PMS oxidation was conducted according to verification and an experimental value obtained is $95.4 \%$. The optimized sulfur removal is $96.7 \%$ according to RSM. The result implies that the experimental value obtained is close to the value calculated from the model and the error rate is only $1.3 \%$, which consequently verifies the model capability. 
TABLE 4: Optimization criteria for sulfur removal.

\begin{tabular}{lc}
\hline Reaction condition & Value \\
\hline PMS/g & 1.6 \\
IL/g & 3.2 \\
Temperature $/{ }^{\circ} \mathrm{C}$ & 46 \\
Time/min & 23 \\
Predicted sulfur removal/\% & 96.7 \\
Experimental sulfur removal/\% & 95.4 \\
\hline
\end{tabular}

\section{Conclusion}

A functional $[\mathrm{Bmim}] \mathrm{Cl} / \mathrm{CoCl}_{2}$ ionic liquid (IL) together with PMS exhibited a high sulfur removal. The quadratic relationship between benzothiophene removal and four experimental conditions was built up based on Box-Behnken Design (BBD) using RSM with 0.9898 fitting coefficient. The optimum conditions were $1.6 \mathrm{~g}$ (20\% wt) PMS solution, $3.2 \mathrm{~g}$ $[\mathrm{Bmim}] \mathrm{Cl} / \mathrm{CoCl}_{2}, 46^{\circ} \mathrm{C}$, and $23 \mathrm{~min}$ with $96.7 \%$ predicted sulfur removal and $95.4 \%$ experimental sulfur removal. The order of influence of experimental conditions is temperature $>$ time $>[\mathrm{Bmim}] \mathrm{Cl} / \mathrm{CoCl}_{2}$ dosage $>$ PMS solution dosage.

\section{Conflicts of Interest}

The authors declare that they have no conflicts of interest.

\section{Acknowledgments}

This work was supported by the Natural Science Foundation of the Henan Province (no. 162300410083) and the National Natural Science Foundation of China (no. 21006057).

\section{References}

[1] S. Xun, W. Zhu, D. Zheng et al., "Synthesis of metal-based ionic liquid supported catalyst and its application in catalytic oxidative desulfurization of fuels," Fuel, vol. 136, pp. 358-365, 2014.

[2] OPEC, World Oil Outlook, Organization of the Petroleum Exporting Countries (OPEC), 2014.

[3] X. Liu, J. Li, and R. Wang, "Study on the desulfurization performance of hydramine/ionic liquid solutions at room temperature and atmospheric pressure," Fuel Processing Technology, vol. 167, pp. 382-387, 2017.

[4] A. Xu, X. Guo, and R. Xu, "Understanding the dissolution of cellulose in 1-butyl-3-methylimidazolium acetate+DMAc solvent," International Journal of Biological Macromolecules, vol. 81, pp. 1000-1004, 2015.

[5] A. Xu, L. Cao, and B. Wang, "Facile cellulose dissolution without heating in [C4mim] $[\mathrm{CH} 3 \mathrm{COO}] / \mathrm{DMF}$ solvent," Carbohydrate Polymers, vol. 125, pp. 249-254, 2015.

[6] L. Zhang, J. Wang, Y. Sun, B. Jiang, and H. Yang, "Deep oxidative desulfurization of fuels by superbase-derived Lewis acidic ionic liquids," Chemical Engineering Journal, vol. 328, pp. 445-453, 2017.

[7] M. Safa, B. Mokhtarani, H. R. Mortaheb, K. Tabar Heidar, A. Sharifi, and M. Mirzaei, "Oxidative Desulfurization of Diesel
Fuel Using a Brønsted Acidic Ionic Liquid Supported on Silica Gel," ENERGY \& FUELS, vol. 31, no. 9, pp. 10196-10205, 2017.

[8] Y. Wang, K. Chen, L. Mo, J. Li, and J. Xu, "Optimization of coagulation-flocculation process for papermaking-reconstituted tobacco slice wastewater treatment using response surface methodology," Journal of Industrial and Engineering Chemistry, vol. 20, no. 2, pp. 391-396, 2014.

[9] X. Chen, D. Song, C. Asumana, and G. Yu, "Deep oxidative desulfurization of diesel fuels by Lewis acidic ionic liquids based on 1-n-butyl-3-methylimidazolium metal chloride," Journal of Molecular Catalysis A: Chemical, vol. 359, pp. 8-13, 2012.

[10] Y. S. Chi, C. P. Li, Q. Z. Jiao, Q. S. Liu, P. F. Yan, and X. M. Liu, "Desulfurization by oxidation combined with extraction using acidic room-temperature ionic liquids," Green Chemistry, vol. 12, pp. 1224-1229, 2011.

[11] K. Cruz-González, O. Torres-Lopez, A. M. García-León, E. Brillas, A. Hernández-Ramírez, and J. M. Peralta-Hernández, "Optimization of electro-Fenton/BDD process for decolorization of a model azo dye wastewater by means of response surface methodology," Desalination, vol. 286, pp. 63-68, 2012.

[12] D. B. Hasan, A. R. Abdul Aziz, and W. M. A. W. Daud, "Oxidative mineralisation of petroleum refinery effluent using Fentonlike process," Chemical Engineering Research and Design, vol. 90, no. 2, pp. 298-307, 2012.

[13] I. Arslan-Alaton, G. Tureli, and T. Olmez-Hanci, "Treatment of azo dye production wastewaters using Photo-Fenton-like advanced oxidation processes: Optimization by response surface methodology," Journal of Photochemistry and Photobiology A: Chemistry, vol. 202, no. 2-3, pp. 142-153, 2009.

[14] S. Sharma, A. Malik, and S. Satya, "Application of response surface methodology (RSM) for optimization of nutrient supplementation for Cr (VI) removal by Aspergillus lentulus AML05," Journal of Hazardous Materials, vol. 164, no. 2-3, pp. 1198-1204, 2009.

[15] H. Li, S. Zhou, Y. Sun, and J. Lv, "Application of response surface methodology to the advanced treatment of biologically stabilized landfill leachate using Fenton's reagent," Waste Management, vol. 30, no. 11, pp. 2122-2129, 2010.

[16] Q. K. Beg, V. Sahai, and R. Gupta, "Statistical media optimization and alkaline protease production from Bacillus mojavensis in a bioreactor," Process Biochemistry, vol. 39, no. 2, pp. 203-209, 2003.

[17] O. Prakash, M. Talat, S. H. Hasan, and R. K. Pandey, "Factorial design for the optimization of enzymatic detection of cadmium in aqueous solution using immobilized urease from vegetable waste," Bioresource Technology, vol. 99, no. 16, pp. 7565-7572, 2008.

[18] J. Madhavan, P. Maruthamuthu, S. Murugesan, and S. Anandan, "Kinetic studies on visible light-assisted degradation of acid red 88 in presence of metal-ion coupled oxone reagent," Applied Catalysis B: Environmental, vol. 83, no. 1-2, pp. 8-14, 2008.

[19] K. H. Chan and W. Chu, "Degradation of atrazine by cobaltmediated activation of peroxymonosulfate: Different cobalt counteranions in homogenous process and cobalt oxide catalysts in photolytic heterogeneous process," Water Research, vol. 43, no. 9, pp. 2513-2521, 2009.

[20] Y. Ji, C. Dong, D. Kong, and J. Lu, "New insights into atrazine degradation by cobalt catalyzed peroxymonosulfate oxidation: Kinetics, reaction products and transformation mechanisms," Journal of Hazardous Materials, vol. 285, pp. 491-500, 2015. 

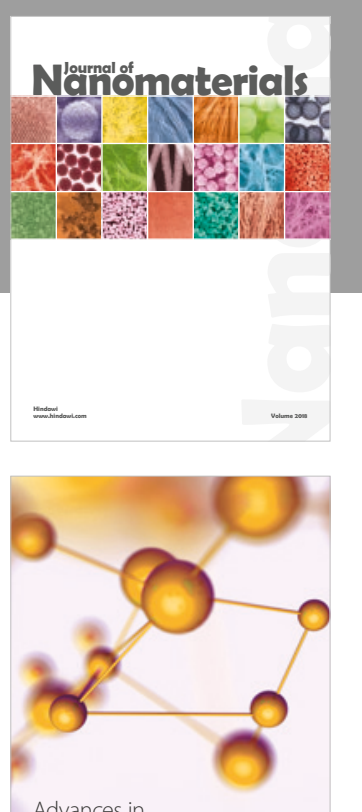

Physical Chemistry
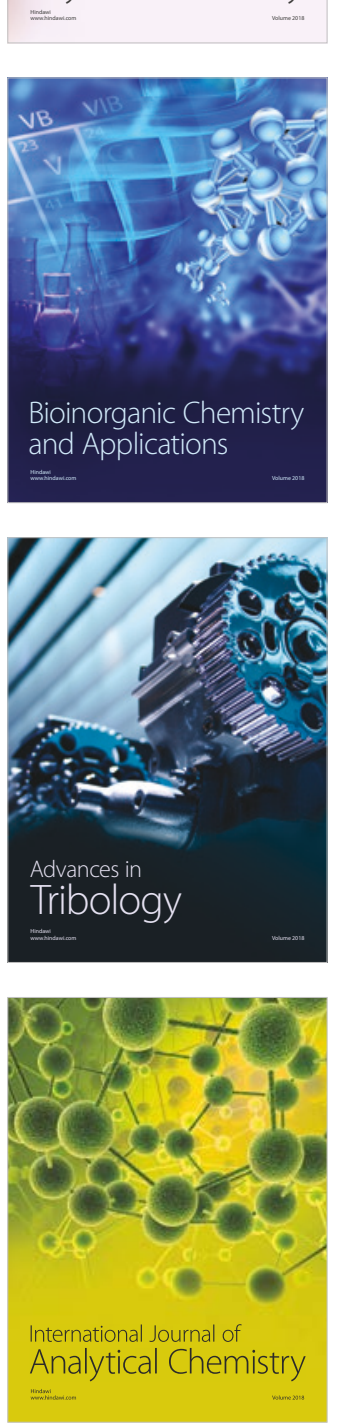

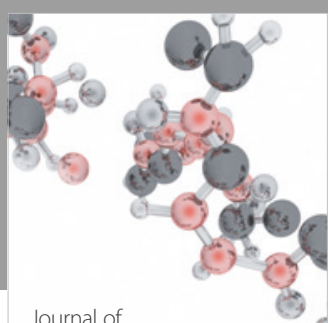

Analytical Methods

in Chemistry

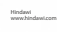

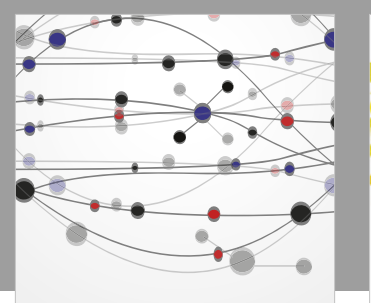

The Scientific World Journal

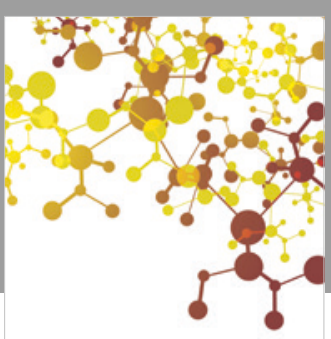

Journal of

Applied Chemistry
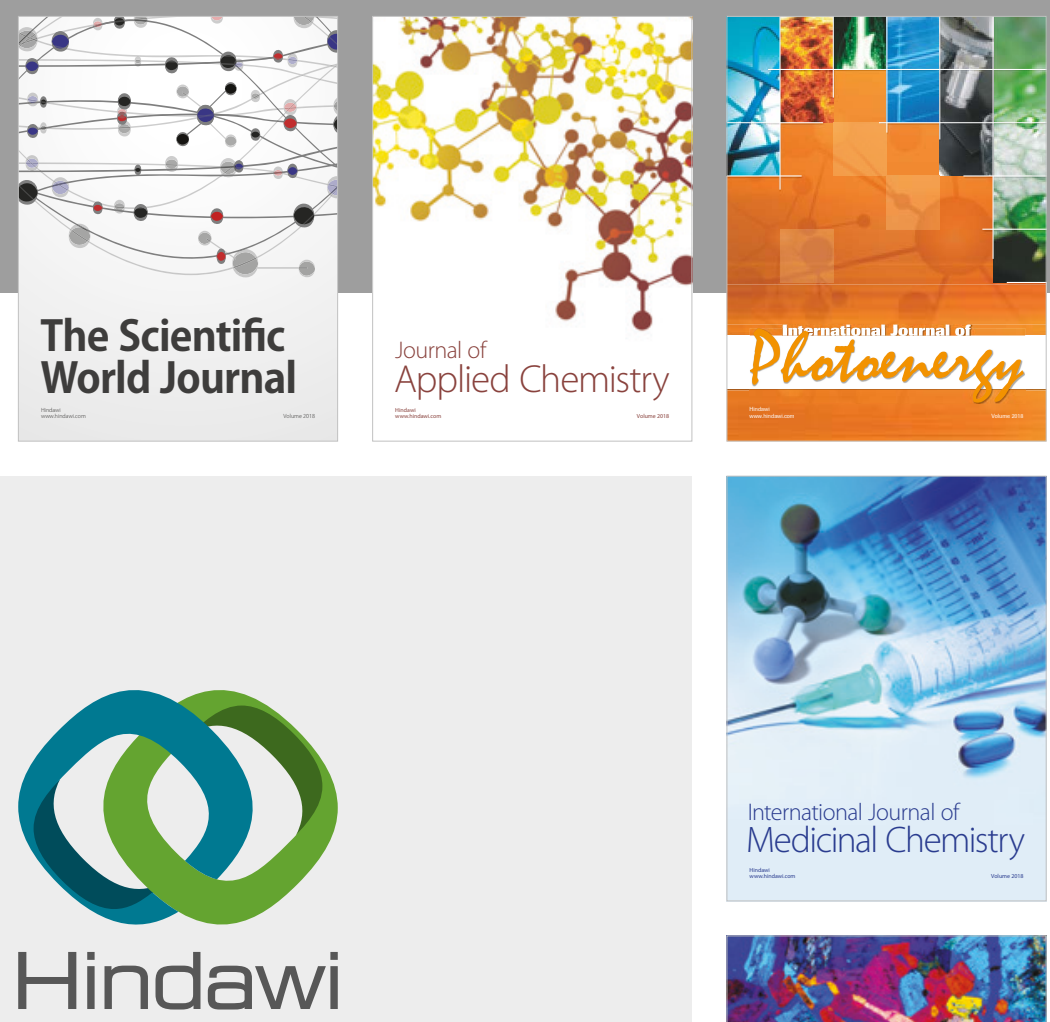

Submit your manuscripts at

www.hindawi.com
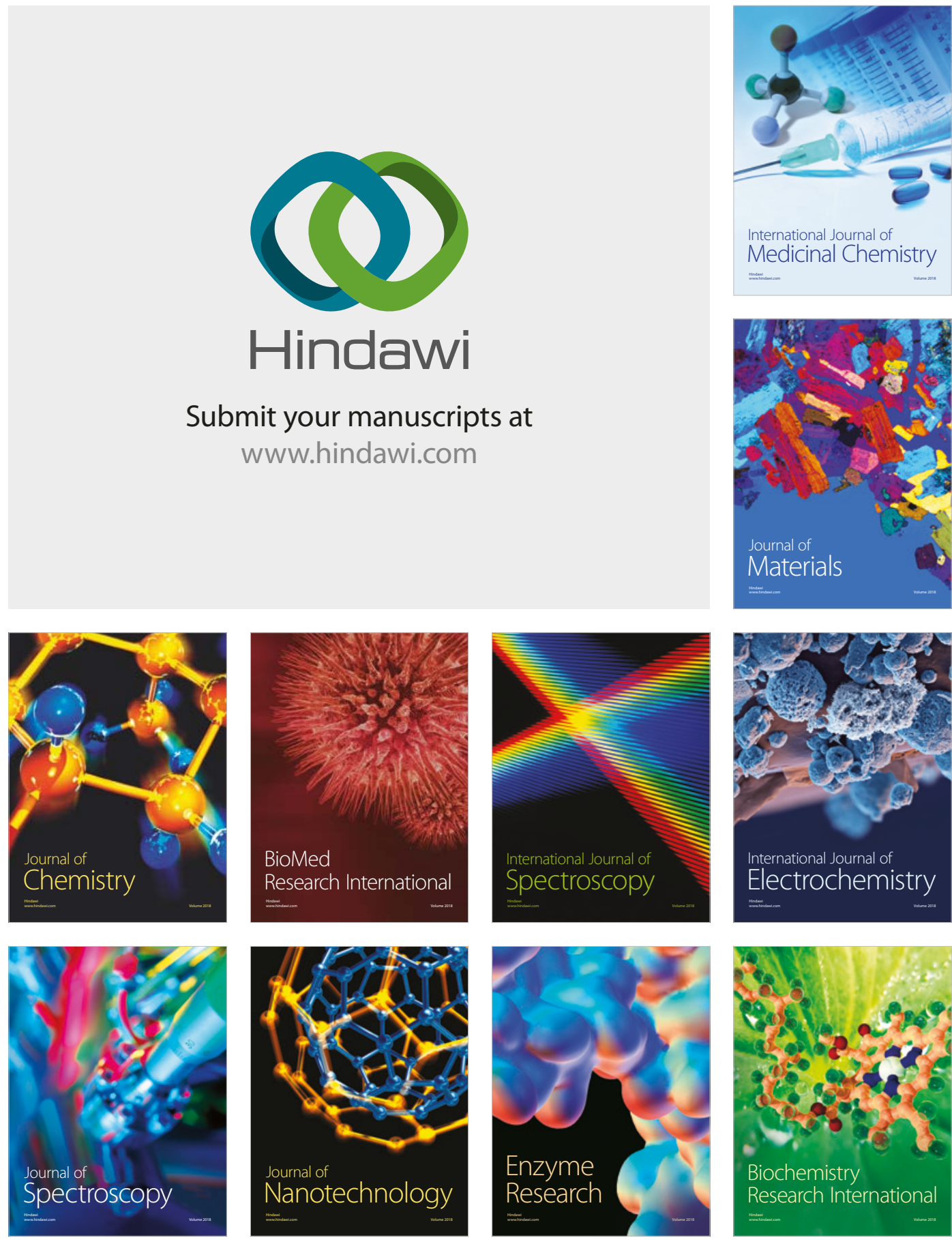
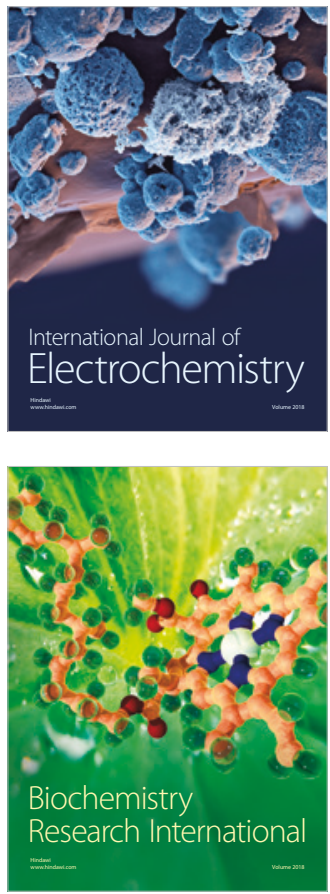